Check for updates

Cite this: RSC Adv., 2019, 9, 31406

\title{
Top-down strategy synthesis of fluorinated graphdiyne for lithium ion battery $\dagger$
}

\author{
Huifang Kang, ${ }^{a}$ Yue Chen, ${ }^{a}$ Lanqing $X u,{ }^{* b}$ Yuda Lin, ${ }^{a}$ Qian Feng, (D) a Hurong Yao ${ }^{a}$ \\ and Yongping Zheng (D) *acd
}

As a novel carbon allotrope, graphdiyne exhibits excellent electrochemical properties such as high specific capacities, outstanding rate performances, and long cycle lives. These properties are attributed to its spand $\mathrm{sp}^{2}$-hybridized bonding and a natural large pore structure. Doping with light elements is a facile way to improve the electrochemical performance of graphdiyne. Herein, we report the preparation of fluorine-doped graphdiyne by exposure to $\mathrm{XeF}_{2}$ under a mild temperature. Compared to pristine graphdiyne, the capacities are doubled. We obtained reversible capacities of fluorinated graphdiyne up to $1080 \mathrm{~mA} \mathrm{~h} \mathrm{~g}^{-1}$ after 600 cycles at a current density of $500 \mathrm{~mA} \mathrm{~g}^{-1}$. At a higher current density of $1000 \mathrm{~mA} \mathrm{~g}^{-1}$, it still retained a high specific capacity of $693 \mathrm{~mA} \mathrm{~h} \mathrm{~g} \mathrm{~g}^{-1}$ after 1000 cycles. Using in situ quantitative nanomechanical probe atomic force microscopy, we further analyzed the surface morphologies and elastic modulus to understand the mechanism of the electrochemical improvement. The fluorinated graphdiyne elastic modulus is doubled in contrast to pristine graphdiyne. The performance improvements are attributed to the improvement in conductivity and enhancement of the mechanical properties.

Received 1st August 2019

Accepted 10th September 2019

DOI: 10.1039/c9ra05974a

rsc.li/rsc-advances energy, catalysis, optoelectronics and other fields. ${ }^{5-9}$ It has been reported that GDY can be used as a high-efficiency lithium storage material, exhibiting good chemical properties, high specific capacity, excellent rate performance and a long cycle. ${ }^{10,11} \mathrm{Li}^{+}$intercalation reaction in graphite occurs only at the edge and defect sites through the basal plane as $\mathrm{LiC}_{6} \cdot{ }^{12}$ In contrast, GDY has 18-C hexagon pores and a layered structure which enable $\mathrm{Li}$ atoms to diffuse with high efficiency both inplane and out-of-plane as $\mathrm{LiC}_{3}$. Theoretically, the predicted capacities for GDY reach to $744 \mathrm{~mA} \mathrm{~h} \mathrm{~g}^{-1} \cdot{ }^{\mathbf{1 3 , 1 4}}$ Experimentally, GDY was reported to possess high reversible capacities up to $520 \mathrm{~mA} \mathrm{~h} \mathrm{~g}^{-1}$ after 400 cycles at a current density of $500 \mathrm{~mA} \mathrm{~g}^{-1}$. The specific capacity is stable at $420 \mathrm{~mA} \mathrm{~h} \mathrm{~g}^{-1}$ even after 1000 cycles under a higher current density of $2 \mathrm{~A} \mathrm{~g}^{-1} \cdot{ }^{11}$

Although GDY as an anode exhibits remarkable capacities and rate performance, the capacity of GDY is still not desirable. To meet the requirements in high power density applications, many efforts have been made to enhance the energy density and specific capacity of GDY-based LIBs, such as basenanocrystallization and light elements doping methods. ${ }^{15-19}$ The doping method was considered as a simple and effective way to improve electrochemical performance in GDY. Two doping technologies have been reported, including in situ doping and mixing doping. As a bottom-up strategy, in situ doping is firstly doping hexaethylbenzene with $\mathrm{H}, \mathrm{Cl}, \mathrm{F}$ atoms and then synthesizing the doped GDY by cross-coupling reaction. ${ }^{17-19}$ Mixing doping is a top-down strategy by mixing GDY with other materials such as ammonium, boron oxide,
${ }^{a}$ College of Physics and Energy, Fujian Normal University, Fujian Provincial Key China. E-mail: zyp@fjnu.edu.cn

${ }^{b}$ College of Photonic and Electronic Engineering, Fujian Normal University, Fuzhou, 350117, China.E-mail: lanqingxu@fjnu.edu.cn

${ }^{c}$ Fujian Provincial Collaborative Innovation Center for Optoelectronic Semiconductors and Efficient Devices, Xiamen, 361005, China

${ }^{d}$ Laboratory of Solid State Microstructures, Nanjing University, Nanjing 210093, China

$\dagger$ Electronic supplementary information (ESI) available. See DOI: 10.1039/c9ra05974a 
ammonium fluoride and synthesizing under thermal annealing, heteroatom doped GDY can be obtained. ${ }^{16,20}$ The advantage of the bottom-up method is the doping elements can be doped in a uniform manner and form a large hexagonal pore to store up Li. The uniformity can extraordinarily improve the electrochemical performance. ${ }^{17-19}$ However, this method changes the GDY intrinsic structure, and so changes its intrinsic properties. Compared to the in situ doping method, the mixing method keeps the GDY intrinsic structure making it more economic and easy to implement. It has been reported that nitrogen doping can be an effective mixing doping way to increase the capacity and cycle performance. ${ }^{16}$ As we know, fluoridation can provide maximum charge polarization to enhance energy-related electrochemical activity and stability owing to fluorine's higher electronegativity compared to nitrogen. Meanwhile, fluoridation can enhance the mechanical properties in carbon-based materials..$^{21,22}$ The top-down strategy fluorine doping of GDY is expected to enhance its mechanical properties to improve the electrochemical performance as lithium storage materials.

Herein, we report facile fluorine doped graphdiyne synthesized by mixing GDY and $\mathrm{XeF}_{2}$ and reacting at a mild temperature. Electrochemical testing results show that the $\mathrm{F}$ doped GDY electrode exhibits excellent electrochemical properties, including higher reversible capacity and outstanding rate performance, compared to those of the pristine GDY electrode. We measured the elastic modulus using an AFM microscope through Peak Force QNM mode to analyse the mechanical changes after doping. As a result, the fluoridation can double the elastic modulus. The mechanical enhancement and conductivity improvements mean the F doped GDY achieves a highly improved specific capacity of $1080 \mathrm{~mA} \mathrm{~h} \mathrm{~g}{ }^{-1}$ at a current density of $500 \mathrm{~mA} \mathrm{~h} \mathrm{~g}^{-1}$. After 1000 cycles, there is still a specific capacity of $693 \mathrm{~mA} \mathrm{~h} \mathrm{~g}^{-1}$ at a current of $1000 \mathrm{~mA} \mathrm{~g}^{-1}$.

\section{Experimental section}

\subsection{Preparation of GDY films}

Firstly, a piece of copper foil $\left(2 \times 8 \mathrm{~cm}^{2}\right)$ was cleaned with $10 \mathrm{M}$ hydrochloric acid $(50 \mathrm{ml})$ for 5 minutes by sonication. Then it was further sonicated by water, ethanol and acetone sequentially for 5 minutes. The after-cleaned foil was dried under argon. Before reaction, the copper foil and pyridine $(170 \mathrm{ml})$ were placed in a three-necked flask and heated at a temperature of $120{ }^{\circ} \mathrm{C}$ under an argon protected atmosphere. Then the temperature was steadily reduced to $80{ }^{\circ} \mathrm{C}$. The hexakis[(trimethylsilyl)ethynyl]benzene $(115 \mathrm{mg})$ was dissolved in a tetrahydrofuran solution $(70 \mathrm{ml})$ at low temperature $\left(0{ }^{\circ} \mathrm{C}\right)$, then tetrabutylammonium fluoride solution (TBAF) $(1.8 \mathrm{ml})$ was added into the solution. The mixture was stirred for 15 minutes and the color of the solution gradually turned purple after adding TBAF. The reaction mixture was diluted with ethyl acetate, and washed with saturated sodium chloride solution sequentially. To remove the additional water, the solution was dried with anhydrous sodium sulfate. After evaporation in vacuo, the residue was dissolved dropwise into the mixed solution containing pyridine. The dropwise process lasts for 8 hours. Then the solution was reacted for more than $48 \mathrm{~h}$. The entire synthesis process lasted approximately 3 days. The obtained samples were washed with hot $\left(60^{\circ} \mathrm{C}\right)$ dimethylformamide (DMF), acetone and ethanol, respectively. Finally, a GDY film was obtained by vacuum drying at $80{ }^{\circ} \mathrm{C}$ for $12 \mathrm{~h}$.

\subsection{Synthesis of fluorine-doped GDY films}

The synthesized GDY $\left(2 \times 8 \mathrm{~cm}^{2}\right)$ was cut into several pieces $(2$ $\times 1.3 \mathrm{~cm}^{2}$ ) and placed in a $50 \mathrm{ml}$ argon-filled polyvinyl chloride reactor along with a quantity of $\mathrm{XeF}_{2}$. It was heated at $180^{\circ} \mathrm{C}$ for $12 \mathrm{~h}$, then cooled down to room temperature. In order to assemble a coin cell, the synthesized F doped GDY film was carefully scraped off one side to expose the copper base as binder. Then it was washed with acetone and ethanol in turn, and dried at $80^{\circ} \mathrm{C}$ for $12 \mathrm{~h}$ in a vacuum.

\subsection{Characterization}

The surface morphologies of F doped GDY and GDY were observed using a scanning electron microscope (SEM, JSM7500F, Japan). Raman spectra were obtained using a LABRAMHRmicro-Raman system (Longjumeau, Paris, France). The chemical compositions of the samples were measured by X-ray photo-electron spectroscopy (XPS, ESCALAB 250, VG, USA), and also confirmed by Fourier Transform Infrared Spectroscopy (FTIR, Thermo-Fisher Nicolet iS50). The surface environments of the samples were observed by atomic force microscopy (AFM). Modulus measurements were performed using an AFM microscope (Dimension icon, Bruker) through Peak Force QNM mode with ScanAsyst ${ }^{\mathrm{TM}}$ in an argon protected atmosphere.

\subsection{Electrochemical measurements}

Electrochemical experiments were performed in a CR2025 coin cell. No initial binder was needed because the copper foil can be used as an electrode directly. All battery components were assembled in standard glove boxes. A lithium metal foil was used as the counter electrode and reference electrode. The electrolyte was prepared by dissolving $1 \mathrm{M} \mathrm{LiPF}_{6}$ into a $1: 1(\mathrm{v} / \mathrm{v})$ mixture of ethylene carbonate (EC) and dimethyl carbonate (DMC). The galvano charge and discharge cycle performance was measured by the LAND CT2001A battery test system. The cycle performance of the battery was tested at $500 \mathrm{~mA} \mathrm{~g}^{-1}$ and $1000 \mathrm{~mA} \mathrm{~g}^{-1}$; the rate performance was tested at different current densities from $50 \mathrm{~mA} \mathrm{~g}^{-1}$ to $1500 \mathrm{~mA} \mathrm{~g}^{-1}$. In the electrochemical workstation (CHI 660C), cyclic voltammetry was carried out at a voltage ranging from $0.01 \mathrm{~V}$ to $3 \mathrm{~V}$ with a scanning rate of $0.1 \mathrm{mV} \mathrm{s}^{-1}$.

\section{Results and discussion}

The morphologies of the membrane before and after fluoridation were characterized by SEM. As shown in Fig. 1a, the asprepared GDY film is uniform, indicating that the GDY film was continuously grown on the copper foil. The morphology of F doped GDY shown in Fig. 1b is similar to that of the GDY film, which means the original structural layout was well maintained during the F-doping process (ESI Fig. S1 and Table S1†). TEM images further demonstrated the uniform and continuous films 

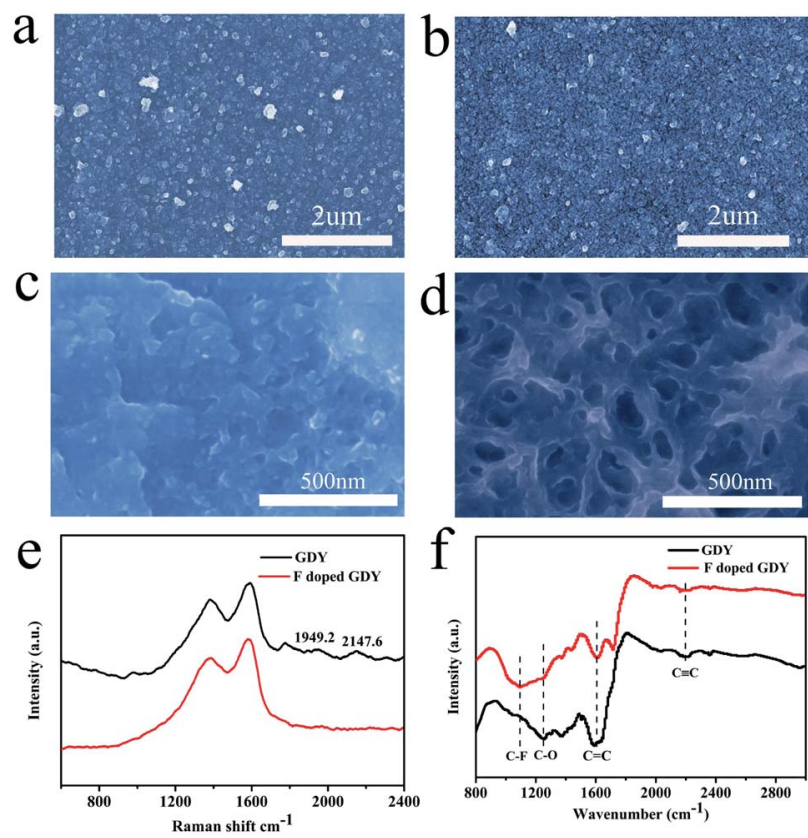

Fig. 1 (a) SEM image morphology of GDY film grown on the surface of copper foil. (b) SEM morphology of the F doped GDY film. Highmagnification SEM of (c) GDY and (d) F doped GDY. (e) Raman spectra of GDY and F doped GDY. (f) FTIR spectra of GDY and F doped GDY film on copper foil.

of GDY and F doped GDY with multilayer structure (ESI Fig. S2 $\dagger$ ). Fig. 1c and d are high-magnification SEM images of GDY and F doped GDY. After F doping, the pore size of the surface is significantly increased. The thicknesses of the GDY and F doped GDY films are approximately $678 \mathrm{~nm}$ and $610 \mathrm{~nm}$, respectively (ESI Fig. S3 $\uparrow$ ). The Raman spectra of the GDY and F doped GDY films are shown in Fig. 1e. It is clear to observe there are four main peaks on both samples. The D band of GDY is located at $1379.6 \mathrm{~cm}^{-1}$, while the D band of $\mathrm{F}$ doped GDY slightly shifts to $1383.5 \mathrm{~cm}^{-1}$, mainly due to structural defects. The G band is located at $1590.2 \mathrm{~cm}^{-1}$ for GDY and $1585.5 \mathrm{~cm}^{-1}$ for $\mathrm{F}$ doped GDY. These peaks correspond to the first-order scattering of the $E_{2 g}$ mode of in-phase stretching vibrations and the breathing vibration of $\mathrm{sp}^{2}$-hybridized carbon domains in aromatic rings, respectively. The ratios of the intensities of the D and G bands $\left(I_{\mathrm{D}} / I_{\mathrm{G}}\right)$ for GDY and F doped GDY are 0.8 and 0.84 , respectively, which indicate both of them are highly ordered. A lower value of $I_{\mathrm{D}} / I_{\mathrm{G}}$ in the F doped GDY case corresponds to more defects and disorder after F doping. ${ }^{4}$ Two other peaks, in the GDY sample the peaks at $2147.6 \mathrm{~cm}^{-1}$ and $1949.2 \mathrm{~cm}^{-1}$, should be the vibration in the acetylenic bond $(-\mathrm{C} \equiv \mathrm{C}-\mathrm{C} \equiv \mathrm{C}-)^{4,16}$ After fluoridation, the alkyne peak becomes weak. The structures of GDY and F doped GDY were further investigated using FTIR as shown in Fig. 1f. The peak of 1500$1650 \mathrm{~cm}^{-1}$ is believed to originate from the skeleton vibration of the aromatic ring. The band at $1251 \mathrm{~cm}^{-1}$ is assigned to the $\mathrm{C}-\mathrm{O}$ stretching. ${ }^{23}$ The weak band observed at $c a .2200 \mathrm{~cm}^{-1}$ is attributed to the acetylene band. The results of FTIR are consistent with previous reports. ${ }^{17,18}$ Due to the C-F stretching vibration and the aromatic bending vibration, an extra peak at $c a .1098 \mathrm{~cm}^{-1}$ can be observed in the $\mathrm{F}$ doped GDY circumstance. ${ }^{24}$

To explore the chemical environment variations after fluoridation, further XPS characterizations were performed. As shown in Fig. 2a, XPS full range spectroscopy of GDY shows that only the $\mathrm{C} 1 \mathrm{~s}$ and $\mathrm{O} 1 \mathrm{~s}$ peaks appear at 284.8 and $532.0 \mathrm{eV}$, respectively. The $\mathrm{O}$ atom was introduced by oxidization during the synthesizing procedure. For the F doped GDY sample, the F 1s peak at $688.0 \mathrm{eV}$ and $\mathrm{F}$ Auger peak at $832.5 \mathrm{eV}$ are seen clearly, showing that the $\mathrm{F}$ atoms were doped successfully. ${ }^{25} \mathrm{Fig}$. $2 \mathrm{~b}$ gives the typical C 1s XPS spectrum of GDY. After subtraction of the Shirley background, followed by fitting with a mixture function of Lorentzian and Gaussian, we could deconvolve the C 1s peak into four main sub-peaks at 284.5, 285.2, 286.9 and $288.5 \mathrm{eV}$, which can be attributed to $\mathrm{C}-\mathrm{C}\left(\mathrm{sp}^{2}\right), \mathrm{C}-\mathrm{C}(\mathrm{sp}), \mathrm{C}-\mathrm{O}$ and $\mathrm{C}=\mathrm{O}$ bonds, respectively. ${ }^{4,26}$ The chemical contents are list in Table 1. The area ratio of $\mathrm{sp} / \mathrm{sp}^{2}$ is 1.59 , which indicates that the diacetyl bond has a better linkage to the benzene ring. ${ }^{4}$ As for $\mathrm{F}$ doped GDY, in addition to the above four peaks mentioned, there are two more peaks at 286.6 and $290.7 \mathrm{eV}$ (Fig. 2c), which correspond to the $\mathrm{C}-\mathrm{F}$ bond and $\mathrm{C}-\mathrm{F}_{2}$ bond respectively. ${ }^{20,27}$ The area ratio of $\mathrm{sp} / \mathrm{sp}^{2}$ reduces to 1.35 , indicating that $\mathrm{C}-\mathrm{F}_{2}$ bonds are formed by $\mathrm{F}$ atoms bonding with the diacetyl link, resulting in a $3.3 \% \mathrm{C}^{-\mathrm{F}_{2}}$ coverage as shown in Table 1. Further more there is a $3.7 \% \mathrm{C}-\mathrm{F}$ bond coverage, whereas the content of $\mathrm{C}=\mathrm{C}$ bonds reduces from $34 \%$ to $32 \%$, which shows that the $\mathrm{F}$ atoms are also likely to bond with benzene rings, leading to a conversion from $\mathrm{sp}^{2}$ to $\mathrm{sp}^{3}$ bonding structure on the membrane. A schematic illustration of the fluoridation structure is shown in Fig. 2d.

The electrochemical properties of F doped GDY were tested using a CR2025 coin-type half cell. The copper foils are directly used as electrodes. However, the copper foils will be dissolved
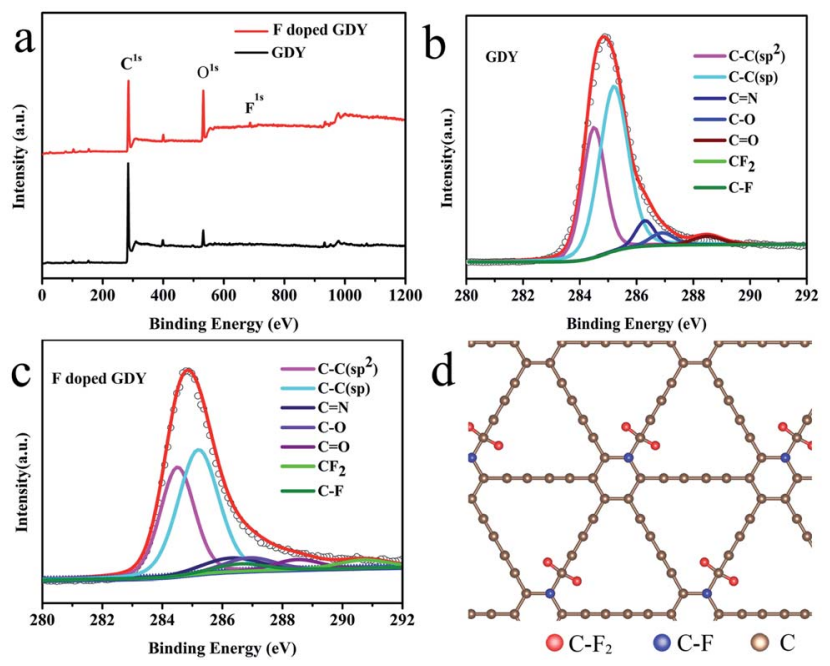

Fig. 2 (a) XPS survey of GDY and F doped GDY over a wide range of binding energies $(0-1200 \mathrm{eV})$. (b) $\mathrm{C}$ 1s spectrum of GDY. (c) C 1s spectrum of F doped GDY. (d) Schematic illustration of F doped GDY structure, red atoms are $F$ bonded with $C$ at ring sites formed as $C-F_{2}$, blue atoms are $F$ bonded with $C$ at chain sites formed as $C-F$. 
Table 1 The chemical compositions of GDY and F doped GDY and deconvolved $O$ and $F$ 1s spectra

\begin{tabular}{lccccc}
\hline Chemical groups & $\mathrm{C}=\mathrm{C}\left(\mathrm{sp}^{2}\right)$ & $\mathrm{C}-\mathrm{C}(\mathrm{sp})$ & $\mathrm{C}-\mathrm{N}$ & $\mathrm{C}-\mathrm{O}, \mathrm{C}=\mathrm{O}$ & $\mathrm{C}-\mathrm{F}$ \\
\hline Location (eV) & 284.5 & 285.2 & 286.3 & $286.9,288.5$ & 286.6 \\
GDY (\%) & 34.0 & 54.3 & 5.4 & 6.3 & 0 \\
F doped GDY (\%) & 32.4 & 43.8 & 6.9 & 9.9 & 0 \\
\end{tabular}

during the reaction process according to the synthetic mechanism of GDY. The mass of active materials was carefully calculated by SEM cross-section characterization and described in ESI Fig. S3 and Table S2. $\dagger$ The cyclic voltammograms (CV) of GDY and F doped GDY are shown in Fig. 3a and $b$ at a scan rate of $0.1 \mathrm{mV} \mathrm{s}^{-1}$. Both of them exhibit an irreversible CV band during the first cathodic scanning. There is no clear reduction in the GDY case consistent with a previous report. ${ }^{11}$ However, F doped GDY showed a significant reduction peak at $0.62 \mathrm{~V}$, an oxidation peak appeared at $0.95 \mathrm{~V}$ and an extra reduction peak appeared at $1.5 \mathrm{~V}$ during the first cathode scan. The previous XPS analysis showed that F doped GDY has $\mathrm{C}-\mathrm{F}$ and $\mathrm{C}-\mathrm{F}_{2}$ bonds after fluoridation. The two reduction peaks correspond to the different $\mathrm{C}-\mathrm{F}$ bonding reductions. After the first cathode scan, the $1.5 \mathrm{~V}$ peak disappeared due to decomposition of the electrolyte and the formation of a solid electrolyte interface (SEI) film. ${ }^{11,16,26}$ From the second cycle, the CV curves are essentially coincident. This indicates that F doped GDY has high reversible charging and discharging behaviors. Fig. $3 \mathrm{c}$ and $\mathrm{d}$ show the charge and discharge curves for GDY and F doped GDY, respectively. The charge and discharge curves for 1, 100, 200, $300,400,500$, and 600 cycles were recorded separately. The specific capacities of the F doped GDY first discharge/charge are $2163 \mathrm{~mA} \mathrm{~h} \mathrm{~g}^{-1}$ and $955 \mathrm{~mA} \mathrm{~h} \mathrm{~g}{ }^{-1}$, respectively. Compared to GDY, the initial coulombic efficiency, cycle life and reversible capacity of F doped GDY are significantly enhanced. The coulombic efficiency of the original GDY (35\%) (ESI Fig. S4†)
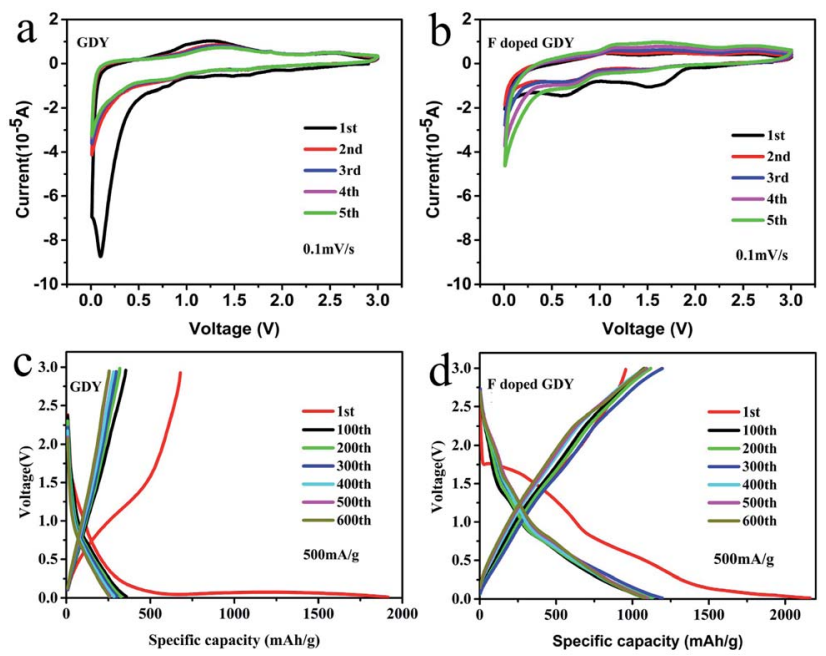

Fig. 3 (a and b) Cyclic voltammetry curves of the GDY and F doped GDY based electrode, scan rate is $0.1 \mathrm{mV} \mathrm{s}^{-1}$. (c and d) Galvanostatic charge/discharge profiles of GDY and F doped GDY electrodes at a current density $500 \mathrm{~mA} \mathrm{~g}^{-1}$, recorded between $0.01 \mathrm{mV}$ and $3 \mathrm{~V}$. was increased to $44 \%$ in the F doped GDY situation (seen in Fig. $4 \mathrm{~b}$ ). The improvement demonstrates that the doping of $\mathrm{F}$ can effectively suppress the decomposition of the electrolyte and the reaction on the surface side to form an SEI film on the F doped GDY electrode. As show in Fig. 3d, the capacity of the 300th discharge was reduced to $1195 \mathrm{~mA} \mathrm{~h} \mathrm{~g}^{-1}$ compared to the first discharge capacity. The reduction can be ascribed to the adsorption of strong lithium ions at specific active sites such C$\mathrm{F}_{2}$ GDY chain sites. After the 600th cycle, the capacity is still above $1080 \mathrm{~mA} \mathrm{~h} \mathrm{~g}^{-1}$, indicating that the F doped GDY material has good capacity retention which is superior to $\mathrm{N}$ doping GDY. ${ }^{16}$

It can be seen from Fig. 4a that F doped GDY exhibits a superior rate performance to GDY within a current density range of 50 to $1500 \mathrm{~mA} \mathrm{~g}^{-1}$. The reversible capacity is about $1600 \mathrm{~mA} \mathrm{~h} \mathrm{~g}^{-1}$ at a current density of $50 \mathrm{~mA} \mathrm{~g}^{-1}$. Even at a high current density of $1500 \mathrm{~mA} \mathrm{~g}^{-1}$, the reversible capacity is still above $500 \mathrm{~mA} \mathrm{~h} \mathrm{~g}^{-1}$. In contrast, the specific capacity of the GDY electrode is $1100 \mathrm{~mA} \mathrm{~h} \mathrm{~g}^{-1}$ at a current density of $50 \mathrm{~mA} \mathrm{~g}^{-1}$ and $300 \mathrm{~mA} \mathrm{~h} \mathrm{~g}^{-1}$ at a current density of $1500 \mathrm{~mA} \mathrm{~g}^{-1}$, respectively. When the current density is reset to $50 \mathrm{~mA} \mathrm{~g}^{-1}, \mathrm{~F}$ doped GDY can still maintain a high reversible capacity of $1400 \mathrm{~mA} \mathrm{~h} \mathrm{~g}^{-1}$, in contrast to a significant shrinking to $761 \mathrm{~mA} \mathrm{~h} \mathrm{~g}^{-1}$ in the GDY case. The excellent rate performance of F doped GDY is suggested to originate from the electronegativity of the $\mathrm{F}$ atoms. F
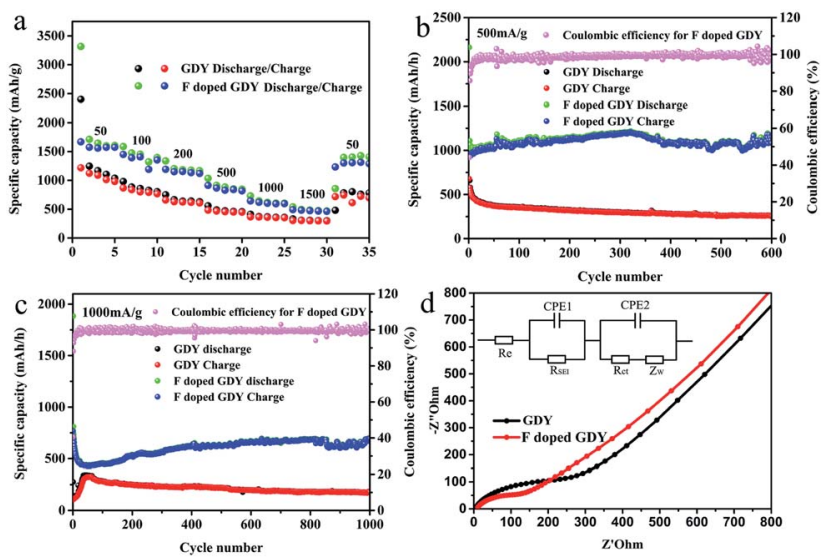

Fig. 4 (a) Different rate performances of $F$ doped GDY and GDY electrodes at varied current densities. (b) Cycle performance of $F$ doped GDY and GDY electrodes under $500 \mathrm{~mA} \mathrm{~g}^{-1}$. (c) Cycle performance of $F$ doped GDY and GDY electrodes under $1000 \mathrm{~mA} \mathrm{~g}^{-1}$. (d) Nyquist plots of GDY and F doped GDY electrodes after 50 cycles under $500 \mathrm{~mA} \mathrm{~g}^{-1}$, and the inset is the equivalent circuit. $R_{\mathrm{e}}$ represents the electrolyte resistance. $R_{\mathrm{SEI}}$ is the resistance of SEI and CPE1 is its capacitance. $R_{\mathrm{ct}}$ is a charge transfer resistor. CPE2 represents a double layer capacitor. $Z_{\mathrm{W}}$ is the impedance of Warburg. 
doping is an effective method to improve the kinetics of lithium diffusion and migration. These phenomena can be better observed in Fig. $4 \mathrm{~b}$ and c. At $500 \mathrm{~mA} \mathrm{~g}^{-1}$ current density, the electrode capacity of $\mathrm{F}$ doped GDY shows a slightly upward trend and reaches to about $1080 \mathrm{~mA} \mathrm{~h} \mathrm{~g}^{-1}$ after 600 cycles. On the contrary, the reversible capacity of GDY gradually decreases to about $300 \mathrm{~mA} \mathrm{~h} \mathrm{~g}^{-1}$ after 600 cycles, which means that $\mathrm{F}$ doped GDY has better stability than that of pristine GDY. As shown in Fig. 4c, when the current density is increased to $1000 \mathrm{~mA} \mathrm{~g}^{-1}$, the reversible capacity of $\mathrm{F}$ doped GDY reaches $693 \mathrm{~mA} \mathrm{~h} \mathrm{~g}^{-1}$ and continues to rise even after 1000 cycles, whereas the reversible capacity of GDY reduces to approximately $200 \mathrm{~mA} \mathrm{~h} \mathrm{~g}^{-1}$ after 1000 cycles. The optimized GDY and $\mathrm{XeF}_{2}$ mass ratio is $1: 16$ (ESI Fig. $\mathrm{S} 5 \dagger$ ). These phenomena show that $\mathrm{F}$ doped GDY has a more stable and larger reversible capacity than that of GDY. It has been reported that the defects on the $\mathrm{N}$-doped graphene sheets provide more $\mathrm{Li}^{+}$storage active sites during the charging/discharging process. ${ }^{28}$ The adsorption energy of $\mathrm{Li}^{+}$is larger and the energy barrier for lithium permeability is lower around the defects, therefore the introduction of $\mathrm{F}$ atoms can increase electron conductivity and generate more hereto atom defects and electrochemically active sites on the GDY membrane. Thus higher capacity was achieved. Moreover, fluorine doping benefits minimization of surface side reactions and formation of a stable interface, thereby improving the electrochemical stability and the reversible capacity of the F doped GDY electrode during the cycling process.

To penetrate more into the depth of the Li storage process after F doping, electrochemical impedance spectroscopy (EIS) measurements were performed. The testing frequency ranges from $0.01 \mathrm{~Hz}$ to $100 \mathrm{kHz}$, and the results after 50 cycles are illustrated in Fig. 4d. The Nyquist diagram shows that F doped GDY has a semicircle diameter smaller than that of the GDY electrode in the high intermediate frequency region, which means that the contact resistance and charge transfer impedance are reduced by F-doping. Different reaction kinetics between GDY and F doped GDY samples were also investigated. The EIS spectra were fitted using the circuit as shown in the inset of Fig. 4d. The electrolyte resistance $R_{\mathrm{e}}$ of GDY and F doped GDY obtained by fitting with the Nyquist plot are listed in Table 2. Both GDY and F doped GDY exhibit similar $R_{\mathrm{e}}(6.28 \Omega$ and $6.14 \Omega$, respectively). However, the SEI resistance of the $\mathrm{F}$ doped GDY electrode $\left(R_{\mathrm{SEI}}=48.88 \Omega\right)$ is much lower than that of GDY $\left(R_{\mathrm{SEI}}=123.10 \Omega\right)$, showing that the doping of the $\mathrm{F}$ atoms can significantly suppress the SEI resistance. The charge transfer resistance of the F doped GDY electrode $\left(R_{\mathrm{ct}}=90.0 \Omega\right)$ is

Table 2 Kinetic parameters of GDY and F doped GDY after 50 cycles under $500 \mathrm{~mA} \mathrm{~g}^{-1}$

\begin{tabular}{lrc}
\hline Samples & GDY & F doped GDY \\
\hline$R_{\mathrm{e}}(\Omega)$ & 6.28 & 6.14 \\
$R_{\mathrm{ct}}(\Omega)$ & 257.80 & 90.0 \\
$R_{\mathrm{SEI}}(\Omega)$ & 123.10 & 48.88
\end{tabular}

also much smaller than that of the GDY electrode $\left(R_{\mathrm{ct}}=257.80\right.$ $\Omega$ ). Owing to F-doping, reduced $R_{\mathrm{SEI}}$ and $R_{\mathrm{ct}}$ are achieved, thus higher conductivity can be expected.

In addition, the high conductivity can promote the electrochemical properties. There have been reports that mechanical enhancement can also improve electrochemical properties previously. ${ }^{29-31}$ Fluorination is an effective method to improve the thermal and mechanical properties within carbon materials. $^{32-34}$ In order to investigate the mechanical changes of GDY and $\mathrm{F}$ doped GDY in the electrochemical process, an in situ quantitative nanomechanical (QNM) atomic force microscope was used to the measure the elastic modulus variations during the first discharge/charge cycle. A commercial silicon nitride tip (RTESPA, cantilever resonance frequency of $200 \mathrm{kHz}$ and nominal elastic constant of $40 \mathrm{~N} \mathrm{~m}^{-1}$ ) with a typical apex radius of $10 \mathrm{~nm}$ was used. Spring constants and resonance frequencies of the cantilevers were both acquired via the thermal-tuning in software. The deflection sensitivity and tip radius were calibrated against standard samples before and after experiments. Maps of the reduced elastic modulus $\left(E_{\mathrm{r}}\right)$ were fitted from the force-distance curves at each pixel through the DerjaguinMuller-Toporov model, ${ }^{35}$

$$
F=\frac{4}{3} \frac{E_{\mathrm{r}}}{1-\nu^{2}} \sqrt{R} \delta^{\frac{3}{2}}
$$

where $F$ and $\delta$ are the force and indentation which can be obtained from the force curve, respectively. $R$ is radius of the tip, $\nu$ is the Poisson's ratio. Here, we chose $\nu=0.2$ to calculate the reduced elastic modulus $E_{\mathrm{r}}$ for the qualitative analysis. All parameters in the formula were dimensionless to enable a visual comparison of the $E_{\mathrm{r}}$ value of the GDY and F doped GDY samples. Galvanostatic cycling was performed using a LAND CT2001A battery test system at $500 \mathrm{~mA} \mathrm{~g}^{-1}$ in a voltage range of 0.01-3 V (Fig. 5a). During the procedure of intercalation (from A to $\mathrm{D}$ ) and deintercalation (from $\mathrm{D}$ to $\mathrm{F}$ ) of lithium, different chemical states including morphologies and elastic modulus phases were recorded and displayed in Fig. 5d. It can be clearly seen from Fig. 5d that the morphologies of both GDY and F doped GDY blur gradually, which can be ascribed the SEI film formation. The elastic modulus are increased during the intercalation process both in GDY and F doped GDY, whereas in reverse during the deintercalation process. To quantize the mechanical variations of intercalation and deintercalation, we calculated the area statistics of the elastic modulus in the dashed square for different states and present them in Fig. 5b and $c$ for comparison. In the case of GDY, the uncycle elastic modulus peak is 4.12. It then increases to 5.03 when the $\mathrm{Li}^{+}$ intercalates into GDY and decreases to 4.29 when it deintercalates. As previous reported, $\mathrm{Li}^{+}$atoms captured by $18-\mathrm{C}$ hexagon prefer to adsorb on the other side to avoid the repulsion with $\mathrm{Li}$ adsorbed over the 6-C hexagon. ${ }^{\mathbf{1 3}, \mathbf{1 4}}$ The aggregation of $\mathrm{Li}^{+}$atoms contribute to enhance the mechanical properties. After deintercalation, the elastic modulus enlarges slightly, owing to the residual $\mathrm{Li}^{+}$atoms bonding with $\mathrm{O}$ defects. The variations in elastic modulus are similar in the F doped GDY case, increasing from 7.92 to 9.57 and then decreasing to 8.93 during discharge/charge. Different to that of GDY, the residual 

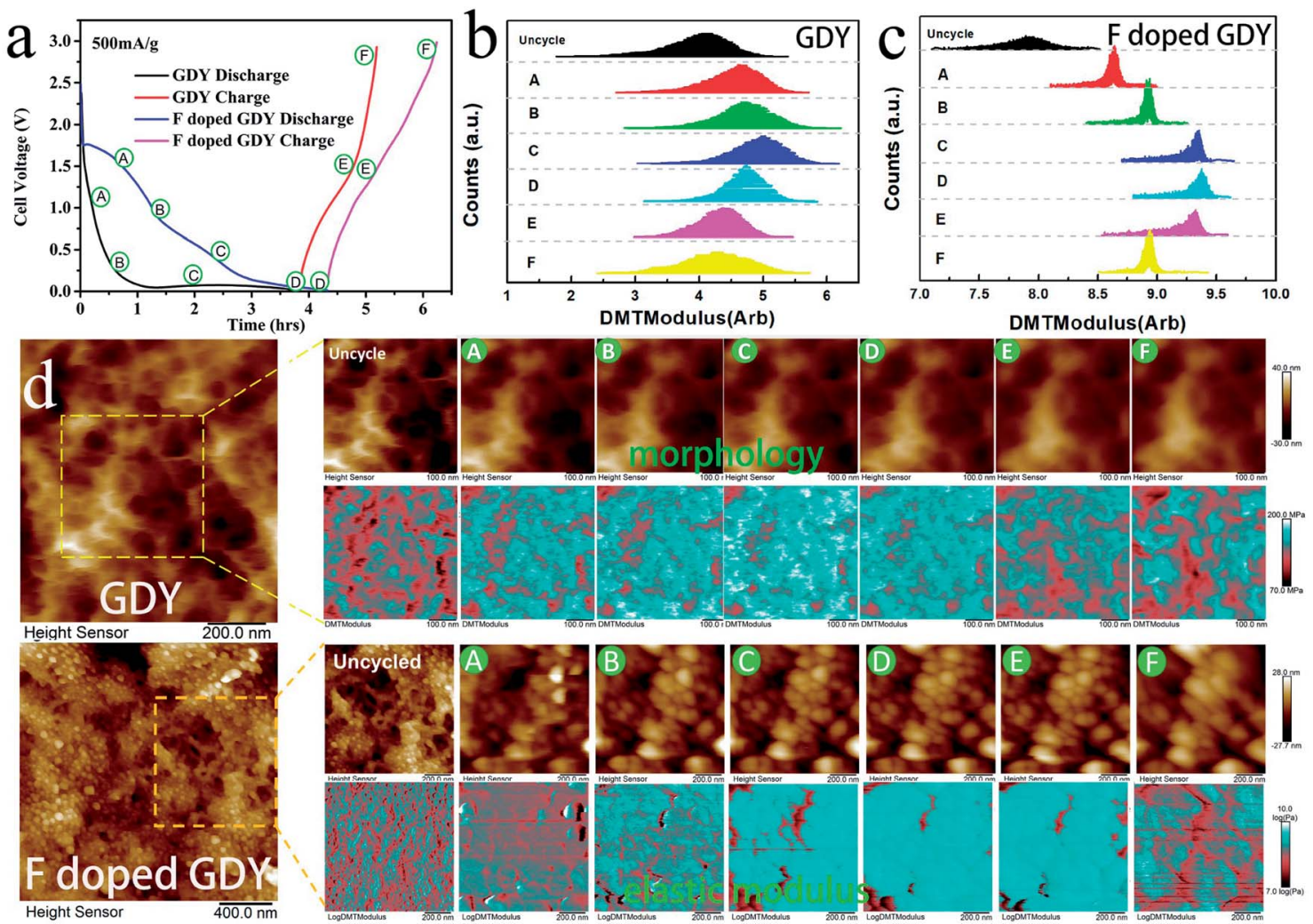
Fig. 5 (a) Galvanostatic cycling of GDY and F doped GDY at $500 \mathrm{~mA} \mathrm{~g}^{-1}$. A to D correspond to the intercalation process and D to F correspond to
the deintercalation process. Area statistics of elastic modulus variations in different states during discharge/charge of (b) GDY and (c) F doped
GDY. (d) In situ Kelvin probe atomic force microscope record of morphology and elastic modulus phase in different chemical states during discharge/charge.

$\mathrm{Li}^{+}$atoms bond not only with $\mathrm{O}$ defects but also with $\mathrm{C}-\mathrm{F}$ bonds, leading to cross-references elastic variation greater than GDY. With a comparison of Fig. $5 b$ and c, we can see that fluoridation almost doubled the elastic modulus of GDY from 4.12 to 7.92. These remarkable mechanical changes contribute to improved reversible capacity and electrochemical stability in F doped GDY during cycling. ${ }^{30}$

\section{Conclusions}

In summary, in this work F doped GDY are facilely synthesized by a mixing GDY and $\mathrm{XeF}_{2}$ reaction at a mild temperature. Various techniques such as SEM, Raman, FTIR and XPS were applied to investigate the morphology changes and electrochemical properties after fluorine doping. The chemical structure results show that $\mathrm{F}$ atoms bond with $\mathrm{C}$ on both benzene rings and diine links. Both samples can used as a negative electrode in lithium-ion batteries to evaluate their lithium storage ability. Compared with pristine GDY, F doped GDY has a better electrochemical performance, such as high specific capacity, excellent rate performance and good cycle life. The current density is $500 \mathrm{~mA} \mathrm{~g}^{-1}$ and the specific capacity is $1080 \mathrm{~mA} \mathrm{~h} \mathrm{~g}^{-1}$. After 1000 cycles, there is still a specific capacity of $693 \mathrm{~mA} \mathrm{~h} \mathrm{~g}^{-1}$ at a current of $1000 \mathrm{~mA} \mathrm{~g}^{-1}$. Thus, the electrochemical performance is significantly improved after fluorine doping. The introduction of $\mathrm{F}$ atoms produces more atom defects and electrochemically active sites contributing to the enhancement in capacity. The remarkable mechanical enhancement within F doped GDY contributes to improving its reversible capacity and electrochemical stability compared to GDY during cycling. Our results show that F doped GDY will serve as a promising anode material for lithium-ion batteries.

\section{Conflicts of interest}

There are no conflicts to declare.

\section{Acknowledgements}

This research was funded by the Natural Science Foundations of China (No. 11404059), the Natural Science Foundations of Fujian Province (Grant No. 2017J01035, No. 2016J01010), the Education and Scientific Research Project for Middle-aged and Young Teachers in Fujian Province (No. JAT170120).

\section{Notes and references}

$1 \mathrm{~K}$. Turcheniuk, D. Bondarev, V. Singhal and G. Yushin, Nature, 2018, 559, 467-470.

2 F. Yao, D. T. Pham and Y. H. Lee, ChemSusChem, 2015, 8, 2284-2311. 
3 G. Wang, X. Shen, J. Yao and J. Park, Carbon, 2009, 47, 20492053.

4 G. Li, Y. Li, H. Liu, Y. Guo, Y. Li and D. Zhu, Chem. Commun., 2010, 46, 3256-3258.

5 X. Zhuang, L. Mao and Y. Li, Electrochem. Commun., 2017, 83, 96-101.

6 Z. Jia, Y. Li, Z. Zuo, H. Liu, C. Huang and Y. Li, Acc. Chem. Res., 2017, 50, 2470-2478.

7 J. Xiao, J. Shi, H. Liu, Y. Xu, S. Lv, Y. Luo, D. Li, Q. Meng and Y. Li, Adv. Energy Mater., 2015, 5, 1401943.

8 Z. Jin, Q. Zhou, Y. Chen, P. Mao, H. Li, H. Liu, J. Wang and Y. Li, Adv. Mater., 2016, 28, 3697-3702.

9 C. Huang, Y. Li, N. Wang, Y. Xue, Z. Zuo, H. Liu and Y. Li, Chem. Rev., 2018, 118, 7744-7803.

10 H. Du, H. Yang, C. Huang, J. He, H. Liu and Y. Li, Nano Energy, 2016, 22, 615-622.

11 C. Huang, S. Zhang, H. Liu, Y. Li, G. Cui and Y. Li, Nano Energy, 2015, 11, 481-489.

12 B. Jungblut and E. Hoinkis, Phys. Rev. B: Condens. Matter Mater. Phys., 1989, 40, 10810.

13 C. Sun and D. J. Searles, J. Phys. Chem. C, 2012, 116, 2622226226.

14 H. Zhang, Y. Xia, H. Bu, X. Wang, M. Zhang, Y. Luo and M. Zhao, J. Appl. Phys., 2013, 113, 044309.

15 H. Shang, Z. Zuo, L. Yu, F. Wang, F. He and Y. Li, Adv. Mater., 2018, 30, 1801459.

16 S. Zhang, H. Du, J. He, C. Huang, H. Liu, G. Cui and Y. Li, ACS Appl. Mater. Interfaces, 2016, 8, 8467-8473.

17 J. He, N. Wang, Z. Cui, H. Du, L. Fu, C. Huang, Z. Yang, X. Shen, Y. Yi, Z. Tu, et al., Nat. Commun., 2017, 8, 1172.

18 N. Wang, J. He, Z. Tu, Z. Yang, F. Zhao, X. Li, C. Huang, K. Wang, T. Jiu, Y. Yi, et al., Angew. Chem., 2017, 129, 10880-10885.

19 J. He, N. Wang, Z. Yang, X. Shen, K. Wang, C. Huang, Y. Yi, Z. Tu and Y. Li, Energy Environ. Sci., 2018, 11, 2893-2903.
20 S. Zhang, Y. Cai, H. He, Y. Zhang, R. Liu, H. Cao, M. Wang, J. Liu, G. Zhang, Y. Li, et al., J. Mater. Chem. A, 2016, 4, 47384744.

21 M. L. Shofner, V. N. Khabashesku and E. V. Barrera, Chem. Mater., 2006, 18, 906-913.

22 M. Abdalla, D. Dean, M. Theodore, J. Fielding, E. Nyairo and G. Price, Polymer, 2010, 51, 1614-1620.

23 Y. Li, C. Guo, J. Li, W. Liao, Z. Li, J. Zhang and C. Chen, Carbon, 2017, 119, 201-210.

24 Y. Song, X. Li, Z. Yang, J. Wang, C. Liu, C. Xie, H. Wang and C. Huang, Chem. Commun., 2019, 55, 6571-6574.

25 Q. Feng, W. Xiao, Y. Liu, Y. Zheng, Y. Lin, J. Li, Q. Ye and Z. Huang, Materials, 2018, 11, 91.

26 S. Zhang, H. Liu, C. Huang, G. Cui and Y. Li, Chem. Commun., 2015, 51, 1834-1837.

27 G. Ross, J. Watts, M. Hill and P. Morrissey, Polymer, 2000, 41, 1685-1696.

28 A. L. M. Reddy, A. Srivastava, S. R. Gowda, H. Gullapalli, M. Dubey and P. M. Ajayan, ACS Nano, 2010, 4, 6337-6342.

29 K. Zhao, W. L. Wang, J. Gregoire, M. Pharr, Z. Suo, J. J. Vlassak and E. Kaxiras, Nano Lett., 2011, 11, 2962-2967.

30 K. Zhao, M. Pharr, S. Cai, J. J. Vlassak and Z. Suo, J. Am. Ceram. Soc., 2011, 94, s226-s235.

31 K. Zhao, G. A. Tritsaris, M. Pharr, W. L. Wang, O. Okeke, Z. Suo, J. J. Vlassak and E. Kaxiras, Nano Lett., 2012, 12, 4397-4403.

32 K. Davami, Y. Jiang, C. Lin, J. Cortes, J. T. Robinson, K. T. Turner and I. Bargatin, RSC Adv., 2016, 6, 11161-11166.

33 X. Wang and P. Wu, ACS Appl. Mater. Interfaces, 2018, 10, 34311-34321.

34 R. R. Nair, W. Ren, R. Jalil, I. Riaz, V. G. Kravets, L. Britnell, P. Blake, F. Schedin, A. S. Mayorov, S. Yuan, et al., Small, 2010, 6, 2877-2884.

35 X. Meng, H. Zhang, J. Song, X. Fan, L. Sun and H. Xie, Nat. Commun., 2017, 8, 1944. 